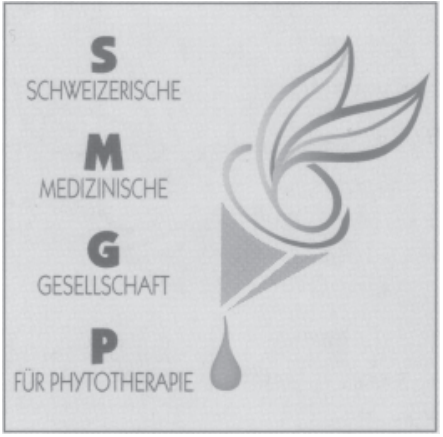

\section{Schweizerische Jahrestagung für Phytotherapie: Phytopharmaka auf Erfolgskurs}

An der diesjährigen Jubiläumsveranstaltung der Schweizerischen Medizinischen Gesellschaft für Phytotherapie (SMGP) fanden sich insgesamt 250 Teilnehmende in Baden ein. Ein facettenreiches Programm erwartete die Zuhörer, die die Bilanz ziehen konnten, dass pflanzliche Arzneimittel den Vergleich mit Synthetica nicht zu scheuen brauchen. Die beachtliche Teilnehmerzahl zeigte einmal mehr das ungebrochene Interesse an der Phytotherapie. Dies kann durchaus als Kontrapunkt zum negativen bundesrätlichen Entscheid bezüglich der Erstattungsfähigkeit der komplementärmedizinischen Therapierichtungen, $z u$ denen auch die Phytotherapie zählt, aufgefasst werden. So wertete auch der Tagungsleiter und Geschäftsführer der SMGP, Prof. Dr. sc. nat. Beat Meier, Hochschule Wädenswil, diese Jubiläumsveranstaltung als eine Fortsetzung der mit der letztjährigen Tagung begonnenen politischen Diskussion. Damals wurden die Ergebnisse des Projekts Evaluation Komplementärmedizin vorgestellt. Prominente, nicht mit der Phytotherapie verbundene Forscher bestätigten damals, dass die Studien mit pflanzlichen Arzneimitteln in den gewählten Indikationen bezüglich Qualität und Resultat vergleichbar sind mit entsprechenden klinischen Studien mit synthetischen Arzneimitteln. Diese Bilanz wurde an der diesjährigen Tagung mit konkreten Beispielen bestätigt.

\section{Johanniskraut mit Vorteilen gleich wirksam wie Synthetica}

Prof. Dr. med. Volker Schulz, Berlin, stellte in seinem Referat Metaanalysen vor, die zeigen, dass das relative Risiko des Non-Response bei den gängigen Zubereitungen aus Johanniskraut (Hypericum perforatum) im Vergleich mit einer Standardtherapie bei mittelschwerer bis leichter Depression ver-

\section{Gesellschaftsmitteilungen · Society Bulletins}

Forsch Komplementärmed Klass Naturheilkd 2005;12:363-364 gleichbar ist. Kritisch wies Schulz darauf hin, dass Studien in Abhängigkeit des geprüften Wirkstoffs unterschiedlich interpretiert und bewertet werden. So wurde eine Studie, in der Johanniskraut gegen Imipramin und Plazebo geprüft wurde, wegen des hohen Plazeboeffekts negativ bewertet. Dagegen wurde eine im Design vergleichbare Studie mit den synthetischen Arzneimitteln Sertralin und Mianserin gegen Plazebo, die ebenfalls einen sehr hohen Plazeboeffekt aufwies, diesbezüglich nicht kritisiert. Gemäss Schulz werde bei diesen Vergleichsstudien zu wenig bedacht, dass die typischen Nebenwirkungen synthetischer Antidepressiva (z.B. Mundtrockenheit) zwangsläufig zu einer Entblindung führen. Dadurch können die Studien gesteuert werden, häufig zu Ungunsten der Phytotherapie. Schulz wies auf ein weiteres Phänomen hin: die Responderrate unter Plazebo hat im Verlauf der letzten 20 Jahre zugenommen. Das Studium der Entwicklung der Antidepressiva zeigt, dass der Massstab des Fortschritts nicht die Verbesserung der Wirksamkeit, sondern die Verbesserung der Verträglichkeit war. Während die Nebenwirkungsquote von trizyklischen Antidepressiva noch bei $30-60 \%$ liegt, beträgt sie bei SSRI (selektive Serotonin-Wiederaufnahmehemmer) nur noch $15-30 \%$. Dem steht bei Johanniskraut in vier besonders gut strukturierten Anwendungsbeobachtungen eine unübertreffbare Quote von $0,1-2,4 \%$ gegenüber. Johanniskraut zeigt aber einige Interaktionen, die zu einer Abnahme der Plasmaspiegel von bestimmten Medikamenten führen, womit sich - wie Schulz ganz anschaulich erwähnte - die so genannte «Blut reinigende» Wirkung von Johanniskraut erklären lässt: «Die Risiken gehen dabei jedoch nicht von Johanniskraut, sondern von der Co-Medikation von Arzneimitteln mit geringer therapeutischer Breite aus.» Bezüglich der Tagestherapiekosten ist Johanniskraut mit den Trizyklika gleichwertig und gegenüber SSRI und anderen um einiges günstiger. Für Schulz ist damit Johanniskraut unter Beachtung aller Aspekte bezüglich Wirksamkeit, Verträglichkeit und Wirtschaftlichkeit die Therapie der Wahl bei leichten bis mittelschweren depressiven Episoden. In der Diskussion wurde die Frage gestellt, inwieweit überhaupt Pharmaka zur Behandlung von solchen Episoden sinnvoll seien. Schulz ist der Meinung, dass die ärztliche Betreuung ein wichtiger Faktor ist, für den Behandlungserfolg jedoch auf Antidepressiva (pflanzlich oder synthetisch) kaum verzichtet werden kann.

\section{Weissdorn und Herzinsuffizienz}

Weissdorn (Crataegus monogyna und andere Crataegus-Arten) wird zur Therapie der Herzinsuffizienz in den Stadien I bis II und inzwischen auch teilweise im Stadium III (gemäss der Klassifikation der New York Heart Association) verwendet. Studien liegen vor allem für Weissdornblätter und -blüten vor. In den letzten Jahren wurden auch Studien mit Präparaten aus Weissdornbeeren durchgeführt. Beide Drogen unterscheiden sich in ihrem Gehalt an Flavonoiden und Procyanidinen. Dr. med. Jörg Melzer, Universitätsspital Zürich, stellte eine Metaanalyse vor, in der von 26 relevanten Studien mit Weissdornblüten und -blättern letztendlich acht randomisierte und plazebo-kontollierte Studien mit zwei Weissdornmonopräparaten eingeschlossen wurden. Als Untersuchungsfaktoren wurden in diesen Studien die maximale Arbeitsbelastung, das Druckfrequenzprodukt und die Belastungstoleranz herangezogen. Ausser für die Belastungstoleranz, bei der nur ein Trend zu beobachten war, ergab sich bezüglich der beiden anderen $\mathrm{Pa}$ rameter unter der Weissdorntherapie eine signifikant bessere Wirksamkeit. Ein systematischer Review zu Präparaten aus Weissdornbeeren zeigte, dass es in einer randomisierten und plazebo-kontrollierten Studie ebenfalls zu einer signifikanten Verbesserung der Belastungstoleranz bei Patienten mit Herzinsuffizienz im Stadium NYHA II kam. Unerwünschte Wirkungen traten unter 
Weissdorn weniger oder gleich häufig wie unter Plazebo auf. Zusätzlich zeigte sich in einer Vergleichsstudie zwischen einem Weissdornpräparat aus Blüten und Blättern und dem ACE-Hemmer Captopril in beiden Gruppen eine signifikante Verbesserung der Arbeitstoleranz bei Patienten mit Herzinsuffizienz im Stadium NYHA II. Zusammengefasst hielt Melzer fest, dass in den Studien die Tagesdosen von 180-1800 mg alkoholische Extrakte eine sehr weite Spanne umfassten. In diesem breiten Dosisbereich kann nun in der Praxis die individuelle Dosierung für den Patienten gefunden werden (meist zwischen 450 und $900 \mathrm{mg}$ ). Mit einer wahrnehmbaren Wirksamkeit ist nach einer Therapiedauer von 3-8 Wochen zu rechnen, was in der Beratung dem Patienten unbedingt mitgeteilt werden muss. Die Behandlungsdauer in Studien lag zwischen 3 und 16 Wochen. Eine erste Langzeitstudie (SPICE) mit einem Crataegus-Extrakt (900 mg/Tag) wird zurzeit durchgeführt. Eine Kombination mit den üblichen synthetischen Herztherapeutika (z.B. Digoxin) scheint gemäss bisheriger Studien möglich. Leider wird derzeit nur sehr wenig über Weissdorn geforscht, obwohl das Potential der Pflanze bei weitem nicht ausgeschöpft scheint.

\section{Arnika versus Ibuprofen}

Andreas Suter, Bioforce AG Roggwil, stellte die altbekannte Heilpflanze Arnica montana vor, die erstmals im 14. Jahrhundert schriftlich erwähnt wurde. Für Arnikablüten gibt es ausführliche Untersuchungen, mit denen der entzündungshemmende Wirkmechanismus aufgeklärt werden konnte. So hemmen vor allem die Sesquiterpenlactone schon in geringen Mengen vollständig den Entzündungsmediator NF- $\mathrm{kB}$, der bei chronischentzündlichen Erkrankungen in hohen Mengen vorkommt. Durch die Hemmung von $\mathrm{NF}-\kappa \mathrm{B}$ werden wiederum Zytokine, Enzyme und Immunrezeptoren gehemmt bzw. blockiert. Dieser Wirkmechanismus ist dem der Cortisone analog. Arnika soll daher nicht eingenommen werden. Erfolgreich verlief eine kürzlich durchgeführte doppelblinde, randomisierte, multizentrische klinische Studie mit einem Arnika-Frischpflanzengel gegen ein 5\%iges Ibuprofen-Gel bei der Behandlung von Fingerpolyarthrose. Die Behandlungsdauer betrug 3 Wochen bei täglich dreimaliger Applikation der Gele. Es konnte gezeigt werden, dass Arnika in allen Zielparametern (u.a. Global Pain und Funktionstüchtigkeit der Hand) Ibuprofen ebenbürtig war. Es fällt auf, dass Ärzte und Patienten die Wirkung des Arnika-Gels besser als die des Ibuprofen-Gels beurteilten. Allerdings kann eine gewisse Entblindung der Studie nicht ausgeschlossen werden.

\section{Cimicifuga und Hormonersatztherapie}

Dr. med. Christine Bodmer, Inselspital Bern, stellte eine Vergleichsstudie eines isopropanolischen Extraktes aus dem Traubensilberkerzenwurzelstock (40 mg/Tag) mit einer niedrig dosierten Hormonersatztherapie $(25 \mu \mathrm{g}$ Estradiol, transdermal plus zyklische Gabe eines Gestagens) zur Behandlung klimakterischer Beschwerden vor. Innerhalb einer 3-monatigen Therapie wurden Hitzewallungen, Schweissausbrüche, Depressionen und Angstzustände sowohl unter Cimicifuga racemosa als auch unter der Östradiolbehandlung signifikant vermindert. In beiden Gruppen wurden auch die Lipide günstig beeinflusst, während die Endometriumdicke unverändert blieb. Cimicifuga hat darüber hinaus eine positive Wirkung auf die Osteoblasten, während Estradiol die Osteoklastentätigkeit senkt, was letztendlich aber zum gleichen Effekt, nämlich der Knochenprotektion, führt. Bezüglich der Haut- und Schleimhautatrophie kam es bei Cimicifuga zu einer knapp nicht signifikanten Verbesserung. Cimicifuga hemmt in vitro das Wachstum bestimmter Brustkrebszelllinien (MCF-7 und MB 231) und fördert die Apoptose von Brustkrebszellen. Eine in der Schweiz gebräuchliche $\mathrm{Zu}-$ bereitung konnte in Kombination mit einer Tamoxifen-Behandlung bei Frauen nach Mammakarzinom die Wallungen reduzieren. Bodmer betonte aber, dass nicht alle Studien mit Cimicifuga eine signifikante Verbesserung der Wallungen und der Depressionen zeigten, sondern oft nur ein deutlicher Trend in Richtung Verbesserung ohne Signifikanz zu beobachten sei. Neueste, teilweise jedoch sehr widersprüchliche Publikationen weisen auf eine mögliche Hemmung von Cytochrom-P450-Enzymen durch Cimicifuga-Extrakte hin. In der Diskussion und im späteren Referat von M. Unger wurde jedoch festgehalten, dass es bis heute keine Hinweise auf eine klinische Relevanz dieser mit viel zu hohen Dosierungen durchgeführten Experimente gibt. Bodmer beurteilte zudem die Kausalität von Berichten zu hepatotoxischen Effekten unter meistens nicht genau geklärten Cimicifuga-Therapien als nicht eindeutig. Bezüglich des Nebenwirkungspotentials ist Cimicifuga günstiger als eine kombinierte Hormontherapie zu beurteilen. Bodmer verwendet in ihrer Praxis sehr oft die pflanzliche Alternative. Sie arbeitet dabei gemäss einem Entscheidungsbaum, der die Wünsche und Einstellungen der Patientin berücksichtigt.

\section{Phytotherapie bei Herpes labialis}

An der Tagung wurden aber nicht nur traditionelle Pflanzen, sondern auch innovative Phytopharmaka in neuen bzw. für Phytotherapeutika eher unüblichen Indikationsgebieten präsentiert. So stellten Dr. med. FranzMartin Janggen, Chur, und Dr. phil. Samuel Büechi, Trogen, ein Präparat gegen Lippenherpes vor, das aus einem Salbei- und einem Rhabarberextrakt besteht. Es ist aufgrund von Ergebnissen eines virologischen Screenings entwickelt worden. Salbeiblätter und Rhabarberwurzel erwiesen sich als antiviral wirksam gegen das Herpes-simplex-Virus Typ 1. Beide Extrakte vereinen Wirkstoffgruppen (Anthrachinone und unterschiedliche Gerbstoffe), die als Komplex eine antivirale Potenz haben. Bei Rhabarber wurde eine Substanz, das Aloeemodin, speziell untersucht. Die Substanz zeigte eine bemerkenswerte IC $_{50}$ von 1,6 respektive $1,5 \mu \mathrm{g} / \mathrm{ml}$ gegenüber den Herpes-simplex-Virus Typ 1 und Typ 2.

In einer prospektiven, randomisierten, kontrollierten, doppelblinden Multizenterstudie wurden die Wirksamkeit und Verträglichkeit des pflanzlichen Kombinationspräparates und des Salbeiextraktes im Vergleich zu einer Aciclovir-Creme untersucht, nachdem sich gezeigt hatte, dass der Rhabarberextrakt allein eine zwar bemerkenswerte, im Vergleich mit der Referenzsubstanz aber keine gleichwertige Wirksamkeit aufwies. Die Untersuchung erfolgte symptomatisch mit Hilfe eines Fragebogens. Das Kombinationspräparat erwies sich als gleichwertig zu Aciclovir. Die reine Salbeicreme war dagegen insgesamt etwas weniger wirksam. Eine Beurteilung der Rezidivhäufigkeit bei den drei Präparaten war leider nicht möglich, da eine grosse Anzahl von Patienten für weitere Beobachtungen nicht zur Verfügung stand. Das Präparat wurde vor kurzem in die Spezialitätenliste aufgenommen. Es erweitert die Möglichkeiten der Phytotherapie um eine wesentliche Indikation. ... (Fortsetzung Heft 1/06)

Beatrix Falch
Korrespondenzadresse:
Geschäftsstelle SMGP
Prof. Dr. Beat Meier
c/o Hochschule Wädenswil
Grüental, Postfach 335
8820 Wädenswil, Schweiz
E-mail b.meier@hsw.ch; www.smgp.ch 